\title{
Ulam-Hyers stability for a nonlinear Volterra integro-differential equation
}

\author{
Ho $\mathrm{Vu}^{1}$ (D), Ngo Van $\mathrm{Hoa}^{2,3 *}$ (10 \\ ${ }^{1}$ Faculty of Mathematical Economics, Banking University of Ho Chi Minh City, Vietnam \\ ${ }^{2}$ Division of Computational Mathematics and Engineering, Institute for Computational Science, Ton Duc \\ Thang University, Ho Chi Minh City, Vietnam \\ ${ }^{3}$ Faculty of Mathematics and Statistics, Ton Duc Thang University, Ho Chi Minh City, Vietnam
}

\begin{abstract}
In this work, the Ulam-Hyers stability and the Ulam-Hyers-Rassias stability for the nonlinear Volterra integro-differential equations are established by employing the method of successive approximation. Some simple examples are given to illustrate the main results.
\end{abstract}

Mathematics Subject Classification (2010). 34K05, 37C75, 45D05

Keywords. Ulam stability, successive approximation, nonlinear Volterra integro-differential equation

\section{Introduction}

Integro-differential equations are a significant research topic from the theoretical point of view as well as of their applications. The reader is referred to the monograph of Volterra [16], Lakshmikantham [11], Medlock [12], the papers [4,6,7] and the references therein. Especially, studying the stability of Ulam-Hyers and Ulam-Hyers-Rassias for differential equations and integro-differential equations has been of great interest (see $[5,8,14,15,17])$ in recent years. In $[5,15]$, by utilizing method of the fixed point theorem, authors presented some kinds of the Hyers-Ulam-Rassias stability to the Volterra integrodifferential equations.

In $[1,2]$, M. Gachpazan and O. Baghani have established the Ulam-Hyers stability for nonlinear integral equation and linear integral equation of the second kind by using successive approximation method. After that with the above idea, Huang et al. [3] proved the Ulam-Hyers stability for delay differential equations of the first order by using successive approximation method. Kucche et al. $[9,10]$ also applied to prove the Ulam-Hyers stability and $E_{\alpha}$-Ulam-Hyers stability results for nonlinear implicit fractional differential equations.

Motivatived by M. Gachpazan and O. Baghani [1,2], and Huang et al. [3], we investigate the Ulam-Hyers stability and Ulam-Hyers-Rassias stability for nonlinear Volterra integrodifferential equations (NVIDE) of the form

\footnotetext{
*Corresponding Author.

Email addresses: ngovanhoa@tdtu.edu.vn (N.V. Hoa), vuh@buh.edu.vn (H. Vu)

Received: 16.11.2018; Accepted: 02.09.2019
} 


$$
\left\{\begin{array}{l}
u^{\prime}(t)=f(t, u(t))+\int_{a}^{t} g(t, s, u(s)) d s, \quad t \in J \\
u(a)=u_{0},
\end{array}\right.
$$

where $J=[a, b], f$ and $g$ are continuous functions.

The rest of this paper is presented as follows: the Ulam-Hyers stability of (1.1) is presented in Section 2, and the Ulam-Hyers-Rassias stability of (1.1) is given in Section 3.

Now, we present the definition of some types of the Ulam-Hyers stability which will be used throughout this paper. First of all, let $\varepsilon>0$ and $\psi \in C\left(J, \mathbb{R}_{+}\right)$. We consider the following inequalities:

$$
\left|v^{\prime}(t)-\mathcal{P}(t)\right| \leq \varepsilon, \quad t \in J
$$

and

$$
\left|v^{\prime}(t)-\mathcal{P}(t)\right| \leq \varepsilon \psi(t), \quad t \in J,
$$

where

$$
\mathcal{P}(t):=f(t, v(t))+\int_{a}^{t} g(t, s, v(s)) d s .
$$

Definition 1.1. The problem (1.1) is Ulam-Hyers stable if there is a constant $K_{f}>0$ such that for each $\varepsilon>0$ and for each solution $v \in C^{1}(J, \mathbb{R})$ of $(1.2)$ there is a solution $u$ of (1.1) satisfying

$$
|v(t)-u(t)| \leq K_{f} \varepsilon .
$$

Definition 1.2. The problem (1.1) is Ulam-Hyers-Rassias stable concerning $\psi \in C\left(J, \mathbb{R}_{+}\right)$ if there is a constant $C_{f}>0$ such that for each $\varepsilon>0$ and for each solution $v \in C^{1}(J, \mathbb{R})$ of (1.3) there is a solution $u$ of (1.1) satisfying

$$
|v(t)-u(t)| \leq C_{f} \varepsilon \psi(t) .
$$

\section{Ulam-Hyers stability for NVIDE (1.1)}

In this section, by employing the successive approximation method, we shall present the Ulam-Hyers stability for NVIDE (1.1).

Remark 2.1. We observe that if the function $v$ is a solution of (1.2), then there is a continuous function $\delta(t)$ on $J$ such that $|\delta(t)| \leq \varepsilon$ and

$$
v^{\prime}(t)=\mathcal{P}(t)+\delta(t) .
$$

Let $f: J \times \mathbb{R} \rightarrow \mathbb{R}$ and $g: J \times J \times \mathbb{R} \rightarrow \mathbb{R}$ are continuous functions. We consider the following hypotheses:

(H1) There exist positive constants $L_{1}, L_{2}$ such that for each $(t, s) \in J \times J$ and $w_{1}, w_{2} \in$ $\mathbb{R}$ one has

$$
\begin{aligned}
\left|f\left(t, w_{1}\right)-f\left(t, w_{2}\right)\right| & \leq L_{1}\left|w_{1}-w_{2}\right|, \\
\left|g\left(t, s, w_{1}\right)-g\left(t, s, w_{2}\right)\right| & \leq L_{2}\left|w_{1}-w_{2}\right| .
\end{aligned}
$$

(H2) Let $\psi \in C\left(J, \mathbb{R}_{+}\right)$in the inequality (1.3). Assume that there exists a constant $C>0$ such that $k C^{k} \leq(b-a) C^{k-1}, \forall k \geq 1$, and $0<C L<1$, and the following hypothesis is satisfied, for $t \in J$,

$$
\int_{a}^{t} \psi(s) d s \leq C \psi(t) .
$$


Theorem 2.2. Assume that $f$ and $g$ satisfy the hypothesis (H1). Then, for each $\varepsilon>0$ if the function $v$ satisfies (1.2), there exists a unique solution $u$ of (1.1) provided $u_{0}=v_{0}$ and $u$ satisfies the following estimate

$$
|u(t)-v(t)| \leq \varepsilon b \exp ((b-a)(1+L)) .
$$

Proof. For each $\varepsilon>0$ and let the function $v$ satisfy (1.2), then basing on Remark 2.1 one has that then there is a continuous function $\delta(t)$ on $J$ such that $|\delta(t)| \leq \varepsilon$ and $v^{\prime}(t)=\mathcal{P}(t)+\delta(t)$. This yields that the function $v$ satisfies the integral equation

$$
v(t)=v_{0}+\int_{a}^{t} \mathcal{P}(s) d s+\int_{a}^{t} \delta(s) d s
$$

where

$$
\int_{a}^{t} \mathcal{P}(s) d s=\int_{a}^{t}\left[f(s, v(s))+\int_{a}^{s} g(s, \tau, v(\tau)) d \tau\right] d s .
$$

We consider the sequence $\left(u_{n}\right)_{n \geq 0}$ defined as follows: $u_{0}(t)=v(t)$ and for $n=1,2, \ldots$

$$
u_{n}(t)=v_{0}+\int_{a}^{t} \mathcal{P}_{n-1}(s) d s,
$$

where

$$
\int_{a}^{t} \mathcal{P}_{n-1}(s) d s=\int_{a}^{t}\left[f\left(s, u_{n-1}(s)\right)+\int_{a}^{s} g\left(s, \tau, u_{n-1}(\tau)\right) d \tau\right] d s .
$$

By (2.2) and (2.3), for $n=1$ one has

$$
\left|u_{1}(t)-u_{0}(t)\right|=\left|v_{0}+\int_{a}^{t} \mathcal{P}_{0}(s) d s-v(t)\right|=\left|\int_{a}^{t} \delta(s) d s\right| \leq \varepsilon(t-a), \forall t \in J .
$$

For $n=1,2, \ldots$, from the hypothesis (H1) one has

$$
\begin{aligned}
\left|u_{n+1}(t)-u_{n}(t)\right| & =\left|\int_{a}^{t} \mathcal{P}_{n}(s) d s-\int_{a}^{t} \mathcal{P}_{n-1}(s) d s\right| \\
& \leq L \int_{a}^{t}\left|u_{n}(s)-u_{n-1}(s)\right| d s+L \int_{a}^{t} \int_{a}^{s}\left|u_{n}(r)-u_{n-1}(r)\right| d r d s,
\end{aligned}
$$

where $L=\max \left\{L_{1}, L_{2}\right\}$. In particular, for $n=1$ and by (2.4) one gets

$$
\begin{aligned}
\left|u_{2}(t)-u_{1}(t)\right| & \leq \varepsilon L \int_{a}^{t}(s-a) d s+\varepsilon L \int_{a}^{t} \int_{a}^{s}(r-a) d r d s \\
& =\varepsilon L\left(\frac{(t-a)^{2}}{2 !}+\frac{(t-a)^{3}}{3 !}\right)
\end{aligned}
$$

and so, for $n=2$, one also obtains

$$
\begin{aligned}
\left|u_{3}(t)-u_{2}(t)\right| & \leq \varepsilon L^{2} \int_{a}^{t}\left(\frac{(s-a)^{2}}{2 !}+\frac{(s-a)^{3}}{3 !}\right) d s+\varepsilon L^{2} \int_{a}^{t} \int_{a}^{s}\left(\frac{(r-a)^{2}}{2 !}+\frac{(r-a)^{3}}{3 !}\right) d r d s \\
& =\varepsilon L^{2}\left(\frac{(t-a)^{3}}{3 !}+2 \frac{(t-a)^{4}}{4 !}+\frac{(t-a)^{5}}{5 !}\right) \\
& \leq 3 \varepsilon L^{2}\left(\frac{(t-a)^{3}}{3 !}+\frac{(t-a)^{4}}{4 !}+\frac{(t-a)^{5}}{5 !}\right)
\end{aligned}
$$

and for $n \geq 4$ we have

$$
\left|u_{n}(t)-u_{n-1}(t)\right| \leq \varepsilon n L^{n-1}\left(\frac{(t-a)^{n}}{n !}+\frac{(t-a)^{n+1}}{(n+1) !}+\ldots+\frac{(t-a)^{2 n}}{(2 n) !}+\frac{(t-a)^{2 n+1}}{(2 n+1) !}\right) .
$$


Then, the estimation (2.5) can be rewritten by:

$$
\begin{aligned}
\left|u_{n}(t)-u_{n-1}(t)\right| & \leq \frac{\varepsilon(t-a)(L(t-a))^{n-1}}{(n-1) !}\left(1+\frac{(t-a)}{n+1}+\frac{(t-a)^{2}}{(n+1)(n+2)}+\ldots\right. \\
& \left.\quad+\frac{(t-a)^{n}}{(n+1)(n+2) \ldots 2 n}+\frac{(t-a)^{n+1}}{(n+1)(n+2) \ldots 2 n(2 n+1)}\right) \\
& \leq \varepsilon b \frac{(L(t-a))^{n-1}}{(n-1) !}\left(1+\frac{(t-a)}{1 !}+\frac{(t-a)^{2}}{2 !}+\ldots+\frac{(t-a)^{n}}{n !}+\frac{(t-a)^{n+1}}{(n+1) !}\right) \\
& \leq \varepsilon b \frac{(L(t-a))^{n-1}}{(n-1) !} \exp (t-a) .
\end{aligned}
$$

Furthermore, if we assume that

$$
\left|u_{n}(t)-u_{n-1}(t)\right| \leq \varepsilon b \frac{(L(t-a))^{n-1}}{(n-1) !} \exp (t-a),
$$

then one also gets

$$
\left|u_{n+1}(t)-u_{n}(t)\right| \leq \varepsilon b \frac{(L(t-a))^{n}}{n !} \exp (t-a), \quad \forall t \in J .
$$

This yields that

$$
\sum_{n=0}^{\infty}\left|u_{n+1}(t)-u_{n}(t)\right| \leq \varepsilon b \exp (b-a) \sum_{n=0}^{\infty} \frac{(L(t-a))^{n}}{n !} .
$$

Since the right-hand series is convergent to the function $\exp (L(t-a))$, for each $\varepsilon>0$ we deduce the series $u_{0}(t)+\sum_{n=0}^{\infty}\left[u_{n+1}(t)-u_{n}(t)\right]$ is uniformly convergent concerning the norm $|\cdot|$ and

$$
\sum_{n=0}^{\infty}\left|u_{n+1}(t)-u_{n}(t)\right| \leq \varepsilon b \exp ((b-a)(1+L))
$$

Assume that

$$
\tilde{u}(t)=u_{0}(t)+\sum_{n=0}^{\infty}\left[u_{n+1}(t)-u_{n}(t)\right]
$$

Then,

$$
u_{j}(t)=u_{0}(t)+\sum_{n=0}^{j}\left[u_{n+1}(t)-u_{n}(t)\right]
$$

is the $j^{\text {th }}$ partial of the series (2.9). From (2.9) and (2.10), we obtain

$$
\lim _{j \rightarrow \infty}\left|\tilde{u}(t)-u_{j}(t)\right|=0, \quad \forall t \in J .
$$

Define $u(t)=\tilde{u}(t), \forall t \in J$. We observe that the limit of the above sequence is a solution to the following equation:

$$
u(t)=v_{0}+\int_{a}^{t} \mathcal{P}(s) d s, \quad \forall t \in J
$$

where

$$
\mathcal{P}(t):=f(t, u(t))+\int_{a}^{t} g(t, s, u(s)) d s
$$


By (2.3), (2.11) and the hypothesis (H1), one has that

$$
\begin{aligned}
\left|u(t)-v_{0}-\int_{a}^{t} \mathcal{P}(s) d s\right| & =\left|\tilde{u}(t)-\left(u_{j}(t)-\int_{a}^{t} \mathcal{P}_{j-1}(s) d s\right)-\int_{a}^{t} \mathcal{P}(s) d s\right| \\
& \leq\left|\tilde{u}(t)-u_{j}(t)\right|+\int_{a}^{t}\left|\mathcal{P}_{j-1}(s)-\mathcal{P}(s)\right| d s \\
& \leq\left|\tilde{u}(t)-u_{j}(t)\right|+L \int_{a}^{t}\left|u_{j-1}(s)-u(s)\right| d s \\
& +L \int_{a}^{t} \int_{a}^{s}\left|u_{j-1}(r)-u(r)\right| d r d s .
\end{aligned}
$$

Combining (2.9) and (2.10), we get

$$
\left|\tilde{u}(t)-u_{j}(t)\right| \leq \sum_{n=j+1}^{\infty}\left|u_{n+1}(t)-u_{n}(t)\right|
$$

and by the estimation (2.7), one has

$$
\left|u(t)-u_{j}(t)\right| \leq \varepsilon b \exp (b-a) \sum_{n=j+1}^{\infty} \frac{(L(t-a))^{n}}{n !}, \quad \forall t \in J .
$$

Hence, it follows from the inequalities (2.12) and (2.13) that

$$
\begin{aligned}
\mid u(t)- & v_{0}-\int_{a}^{t} \mathcal{P}(s) d s \mid \leq \varepsilon b e^{b-a} \sum_{n=j+1}^{\infty} \frac{(L(t-a))^{n}}{n !} \\
& +\varepsilon L b e^{b-a}\left(\int_{a}^{t} \sum_{n=j+1}^{\infty} \frac{(L(s-a))^{n}}{n !} d s+\int_{a}^{t} \int_{a}^{s} \sum_{n=j+1}^{\infty} \frac{(L(r-a))^{n}}{n !} d r d s\right) \\
\leq & \varepsilon b e^{b-a}\left[\sum_{n=j+1}^{\infty} \frac{(L(t-a))^{n}}{n !}+\sum_{n=j+1}^{\infty} L^{n+1}\left(\frac{(t-a)^{n+1}}{(n+1) !}+\frac{(t-a)^{n+2}}{(n+2) !}\right)\right] .
\end{aligned}
$$

Taking limit as $n \rightarrow \infty$, we see that the right-hand series of (2.14) is convergent. Therefore, one deduces that

$$
\left|u(t)-v_{0}-\int_{a}^{t} \mathcal{P}(s) d s\right| \leq 0, \quad \forall t \in J
$$

This means that

$$
u(t)=v_{0}+\int_{a}^{t} \mathcal{P}(s) d s, \quad \forall t \in J,
$$

which is a solution of (1.1). In addition, from the estimation (2.8), we have the estimate as follows:

$$
|u(t)-v(t)| \leq \varepsilon b \exp ((b-a)(1+L)) .
$$

To show the uniqueness of solution to the problem (1.1), we assume that $\widehat{u}(t)$ is another solution of (1.1), which has the form

$$
\widehat{u}(t)=v_{0}+\int_{a}^{t} \widehat{\mathcal{P}}(s) d s, \quad \forall t \in J,
$$

where

$$
\widehat{\mathcal{P}}(t):=f(t, \widehat{u}(t))+\int_{a}^{t} g(t, s, \widehat{u}(s)) d s .
$$

By using the hypothesis (H1), one obtains

$$
\gamma(t) \leq L \int_{a}^{t} \gamma(s) d s+L \int_{a}^{t} \int_{a}^{t} \gamma(r) d r d s, \quad \forall t \in J .
$$


where $\gamma(t):=|u(t)-\widehat{u}(t)|$. Then by applying Gronwall's lemma (see Theorem 2.1 in [13]), we infer that $\gamma(t)=0$ on $J$. So, $u(t)=\widehat{u}(t)$. This completes the proof.

\section{Ulam-Hyers-Rassias stability for NVIDE (1.1)}

In this section, with the same manner as in Section 2, by using the successive approximation method, we also present the Ulam-Hyers-Rassias stability for NVIDE (1.1).

Remark 3.1. We observe that if the function $v$ is a solution of (1.3), then there is a continuous function $\xi(t)$ on $J$ such that $|\xi(t)| \leq \varepsilon \psi(t)$ and

$$
v^{\prime}(t)=\mathcal{P}(t)+\xi(t) .
$$

Theorem 3.2. Assume that the hypotheses (H1) and (H2) are held. Then, for each $\varepsilon>0$, if the function $v$ satisfies (1.3), there is a unique solution $u$ of (1.1) with $u_{0}=v_{0}$, and $u$ satisfies the following estimate, for $t \in J$,

$$
|v(t)-u(t)| \leq \varepsilon \frac{(b-a)}{(1-C)(1-C L)} \psi(t) .
$$

Proof. For each $\varepsilon>0$ let the function $v$ satisfy (1.3), then by basing on Remark 3.1, one has that there is a continuous function $\xi(t)$ on $J$ such that $|\xi(t)| \leq \varepsilon \psi(t)$ and $v^{\prime}(t)=$ $\mathcal{P}(t)+\xi(t)$. This yields that the function $v$ satisfies the integral equation as follows:

$$
v(t)=v_{0}+\int_{a}^{t} \mathcal{P}(s) d s+\int_{a}^{t} \xi(s) d s
$$

where

$$
\int_{a}^{t} \mathcal{P}(s) d s=\int_{a}^{t}\left[f(s, v(s))+\int_{a}^{s} g(s, \tau, v(\tau)) d \tau\right] d s .
$$

Similar to the proof of Theorem 2.2, we also reconsider the sequence $\left(u_{n}\right)_{n \geq 0}$ defined as in (2.3) with $u_{0}(t)=v(t), \forall t \in J$. Now, by (2.3), the hypothesis (H3) and (3.2), for $n=1$ one has

$$
\left|u_{1}(t)-u_{0}(t)\right|=\left|v_{0}+\int_{a}^{t} \mathcal{P}_{0}(s) d s-v(t)\right| \leq \varepsilon \int_{a}^{t} \psi(s) d s \leq \varepsilon C \psi(t), \forall t \in J .
$$

For $n=1,2, \ldots$ and from the hypothesis (H1), one has

$$
\left|u_{n+1}(t)-u_{n}(t)\right| \leq L \int_{a}^{t}\left(\left|u_{n}(s)-u_{n-1}(s)\right| d s+\int_{a}^{s}\left|u_{n}(r)-u_{n-1}(r)\right| d r\right) d s,
$$

where $L=\max \left\{L_{1}, L_{2}\right\}$. In particular, for $n=1$ one has

$$
\left|u_{2}(t)-u_{1}(t)\right| \leq \varepsilon L C \int_{a}^{t} \psi(s) d s+\varepsilon L C \int_{a}^{t} \int_{a}^{s} \psi(r) d r d s=\varepsilon L\left(C^{2}+C^{3}\right) \psi(t), \quad \forall t \in J
$$

and so, for $n=2$, we also obtain

$$
\begin{aligned}
\left|u_{3}(t)-u_{2}(t)\right| & \leq L \int_{a}^{t}\left|u_{2}(s)-u_{1}(s)\right| d s+L \int_{a}^{t} \int_{a}^{s}\left|u_{2}(r)-u_{1}(r)\right| d r d s \\
& \leq 3 \varepsilon L^{2}\left(C^{3}+C^{4}+C^{5}\right) \psi(t) .
\end{aligned}
$$

and for $n \geq 4$ we have

$$
\left|u_{n}(t)-u_{n-1}(t)\right| \leq n \varepsilon\left(C^{n}+C^{n+1}+\ldots+C^{2 n}+C^{2 n+1}\right) L^{n-1} \psi(t) .
$$


Then, by the hypothesis (H3), the estimation (2.5) is rewritten by:

$$
\begin{aligned}
\left|u_{n}(t)-u_{n-1}(t)\right| & \leq \varepsilon(b-a)(C L)^{n-1}\left(1+C^{1}+\ldots+C^{n+1}\right) \psi(t) \\
& \leq \varepsilon(b-a)\left(\frac{1-C^{n+1}}{1-C}\right)(C L)^{n-1} \psi(t), \quad \forall t \in J .
\end{aligned}
$$

In addition, if the assumption

$$
\left|u_{n}(t)-u_{n-1}(t)\right| \leq \varepsilon(b-a)\left(\frac{1-C^{n+1}}{1-C}\right)(C L)^{n-1} \psi(t), \quad \forall t \in J,
$$

is satisfied, then by using the mathematical induction we also get

$$
\left|u_{n+1}(t)-u_{n}(t)\right| \leq \varepsilon(b-a)\left(\frac{1-C^{n+2}}{1-C}\right)(C L)^{n} \psi(t), \quad \forall t \in J
$$

This yields that

$$
\sum_{n=0}^{\infty}\left|u_{n+1}(t)-u_{n}(t)\right| \leq \varepsilon(b-a)\left(\frac{1}{1-C}\right) \sum_{n=0}^{\infty}(C L)^{n} \psi(t)
$$

By the hypothesis (H3), we observe that $\sum_{n=0}^{\infty}(C L)^{n} \rightarrow \frac{1}{1-C L}$ as $n \rightarrow \infty$. Hence, for every $\varepsilon>0$ we infer that the series $u_{0}(t)+\sum_{n=0}^{\infty}\left[u_{n+1}(t)-u_{n}(t)\right]$ is uniformly convergent on $J$ and

$$
\sum_{n=0}^{\infty}\left|u_{n+1}(t)-u_{n}(t)\right| \leq \varepsilon \frac{(b-a)}{(1-C)(1-C L)} \psi(t), \quad \forall t \in J .
$$

With the same manner as in the proof of Theorem 2.2 , we also can show that $u(t)$ is a solution of (1.1) which has form

$$
u(t)=v_{0}+\int_{a}^{t} \mathcal{P}(s) d s, \quad \forall t \in J,
$$

where

$$
\mathcal{P}(t):=f(t, u(t))+\int_{a}^{t} g(t, s, u(s)) d s .
$$

In addition, the following estimate is also satisfied

$$
|u(t)-v(t)| \leq \varepsilon \frac{(b-a)}{(1-C)(1-C L)} \psi(t), \quad \forall t \in J .
$$

Therefore, (1.1) is Ulam-Hyers-Rassias stable.

\section{Examples}

In this section, two simple examples are presented to illustrate our results.

Example 4.1. Consider the following problem

$$
\left\{\begin{array}{l}
u^{\prime}(t)=1+\int_{0}^{t} u(s) d s, \quad \forall t \in[0,1] \\
u(0)=1
\end{array}\right.
$$

We see that $v(t)=1, \forall t \in[0,1]$ complies with the following inequality

$$
\left|v^{\prime}(t)-1-\int_{0}^{t} v(s) d s\right| \leq 2
$$


Now, we can choose $v_{0}(t)=u(0)=1$. By using the successive approximation method as in Theorem 2.2, we obtain the following successive solution to (4.1) as

$$
\begin{aligned}
& v_{0}(t)=1, \\
& u_{1}(t)=v(0)+\int_{0}^{t}\left(1+\int_{0}^{s} u(r) d r\right) d s=1+t+\frac{t^{2}}{2 !} .
\end{aligned}
$$

Then it is no difficult to see that $u(t)=1+t+\frac{t^{2}}{2 !}$ forms a solution (4.1) and one gets the estimate

$$
|v(t)-u(t)|=\left|1-\left(1+t+\frac{t^{2}}{2 !}\right)\right| \leq \frac{3}{2}
$$

Next, we define the function $u^{*}(t)=1+t+\frac{t^{2}}{2 !}+\frac{t^{3}}{3 !}+\frac{t^{4}}{4 !}$ is also a solution of (4.1) and we also have

$$
\left|v(t)-u^{*}(t)\right|=\left|1-\left(1+t+\frac{t^{2}}{2 !}+\frac{t^{3}}{3 !}+\frac{t^{4}}{4 !}\right)\right| \leq \frac{17}{24} .
$$

Therefore, it shows the function $u^{*}(t)$ is better approximate solution than the function $u(t)$.

Example 4.2. Consider the following problem

$$
u^{\prime}(t)=u(t)+\int_{0}^{t} \frac{u(s)}{1+u(s)} d s
$$

where $t \in[0,1]$. We set

$$
f(t, u(t))=u(t) \quad \text { and } \quad g(t, s, u(s))=\frac{u(s)}{1+u(s)} .
$$

Then, we see that

$$
\left|f\left(t, w_{1}\right)-f\left(t, w_{2}\right)\right|=\left|w_{1}-w_{2}\right|
$$

and

$$
\begin{aligned}
\left|g\left(t, s, w_{1}(s)\right)-g\left(t, s, w_{2}(s)\right)\right| & =\left|\frac{w_{1}(s)}{1+w_{1}(s)}-\frac{w_{2}(s)}{1+w_{2}(s)}\right| \\
& \leq \frac{\left|w_{1}-w_{2}\right|}{\left(1+w_{1}\right)\left(1+w_{2}\right)} \\
& \leq\left|w_{1}-w_{2}\right| .
\end{aligned}
$$

This yields that the hypotheses of Theorem (2.2) is satisfied. That means Eq. (4.2) has unique solution on $[0,1]$. Furthermore, if the function $v$ satisfies

$$
\left|v^{\prime}(t)-v(t)-\int_{0}^{t} \frac{v(s)}{1+v(s)} d s\right| \leq \varepsilon
$$

then by basing on the result of Theorem 2.2, there exists a solution $u$ of Eq. (4.2) satisfying

$$
|u(t)-v(t)| \leq \varepsilon \exp (2), \quad \forall t \in[0,1] .
$$

This means that the problem (4.2) is Ulam-Hyers stable.

\section{Conclusion}

The results of Ulam-Hyers stability to the nonlinear Volterra integro-differential equation have been investigated by Janfada et al. [5] and Sevgin et al. [15] by employing the fixed point theorem. In our paper, we present the Ulam-Hyers stability and the Ulam-HyersRassias stability to NVIDE (1.1) by employing the method of successive approximation. 


\section{References}

[1] M. Gachpazan and O. Baghani, Hyers-Ulam stability of nonlinear integral equation, Fixed Point Theory Appl. 2010, 6 pages, 2010.

[2] M. Gachpazan and O. Baghani, Hyers-Ulam stability of volterra integral equation, Inter. J. Nonlinear Anal. Appl. 1 (2), 19-25, 2010.

[3] J. Huang and Y. Li, Hyers-Ulam stability of delay differential equations of first order, Math. Nachr. 289 (1), 60-66, 2016.

[4] H.V. Jain and H.M. Byrne, Qualitative analysis of an integro-differential equation model of periodic chemotherapy, Appl. Math. Lett. 25 (12), 2132-2136, 2012.

[5] M. Janfada and G. Sadeghi, Stability of the Volterra integrodifferential equation, Folia Math. 18 (1), 11-20, 2013.

[6] C. Jin and J. Luo, Stability of an integro-differential equation, Comput. Math. Appl. 57 (7), 1080-1088, 2009.

[7] M. Joshi, An existence theorem for an integro-differential equation, J. Math. Anal. Appl. 62 (1), 114-124, 1978.

[8] S.-M. Jung and J. Brzdek, Hyers-Ulam stability of the delay equation $y^{\prime}(t)=\lambda y(t-\tau)$, Abstr. Appl. Anal. 2010, Art. Id. 372176, 2010.

[9] K.D. Kucche and S.T. Sutar, Stability via successive approximation for nonlinear implicit fractional differential equations, Moroccan J. Pure Appl. Anal. 3 (1), 36-55, 2017.

[10] K.D. Kucche and S.T. Sutar, On existence and stability results for nonlinear fractional delay differential equations, Bol. Soc. Parana. Mat. 36 (4), 55-75, 2018.

[11] V. Lakshmikantham, Theory of Integro-Differential Equations, CRC Press, Boca Raton, Florida, NY, 1995.

[12] J.P. Medlock, Integro-differential equation models in ecology and epidemiology, University of Washington, PhD Thesis, 2004.

[13] J.A. Oguntuase, On an inequality of Gronwall, J. Inequal. Pure Appl. Math. 2 (1), Art. No. 9, 2001.

[14] A.Z. Rahim Shah, A fixed point approach to the stability of a nonlinear volterra integrodifferential equation with delay, Hacet. J. Math. Stat. 47 (3), 615-623, 2018.

[15] S. Sevgin and H. Sevli, Stability of a nonlinear volterra integro-differential equation via a fixed point approach, J. Nonlinear Sci. Appl. 9, 200-207, 2016.

[16] V. Volterra, Theory of Functionals and of Integral and Integro-Differential Equations, Dover, New York, NY, 1959.

[17] A. Zada and S.O. Shah, Hyers-Ulam stability of first-order non-linear delay diffierential equations with fractional integrable impulses, Hacet. J. Math. Stat. 47 (5), 1196-1205, 2018. 